\title{
Last night a DJ went to space
}

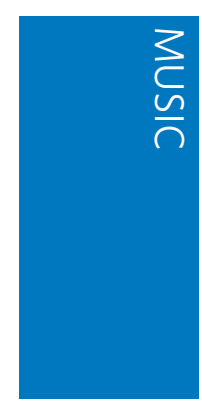

It is uncommon to see a full orchestra lined up on stage next to a pair of turntables. And yet that's exactly what you'll see when you embark on Jeff Mills's cosmic tour, Planets. The renowned techno DJ and composer worked on this 18-piece score between 2006 and 2015, intertwining classical and electronic music, and creating a performance that would send us on a journey through the Solar System. In June, Planets premiered in the UK at the Barbican Centre, London, featuring Jeff Mills and the Britten Sinfonia orchestra ensemble conducted by Christophe Mangou.

The score was inspired by Gustav Holst's seven-movement classical suite The Planets, in which each movement is named after a planet - from Mercury to Neptune. But the similarities between the two scores don't extend much beyond their subject. In addition to the obvious difference in terms of instruments involved, the compositions by Holst and Mills very much mirror their respective historical context, as well as their attitude to space science.

Holst's pieces, which premiered in 1918 , are astrological in concept, and were composed to elicit the emotions with which each planet is associated. The result is a light-hearted and dreamlike travel through the human psyche, rather than actual space. Indeed, the movements don't even respect the real ordering of the planets, and there isn't one for Earth.

Mills's work suggests an era of much more detailed knowledge of space and its processes. The journey starts with an introduction, moves on to Mercury and then explores the whole Solar System. Nine of the pieces correspond to our stops on each planet, including Earth and Pluto (discovered after 1918), and eight more represent the transits between them. What was just absent empty space in Holst's masterwork is now worthy of our attention - as we now know that there is no such thing as emptiness.

The electronic music makes the overall experience feel more complete, not unlike our current knowledge of the Solar System if compared to 1918. But it's really the juxtaposition of the electronic and classical music that makes the pieces so good at immersing us in Mills's tour. It's

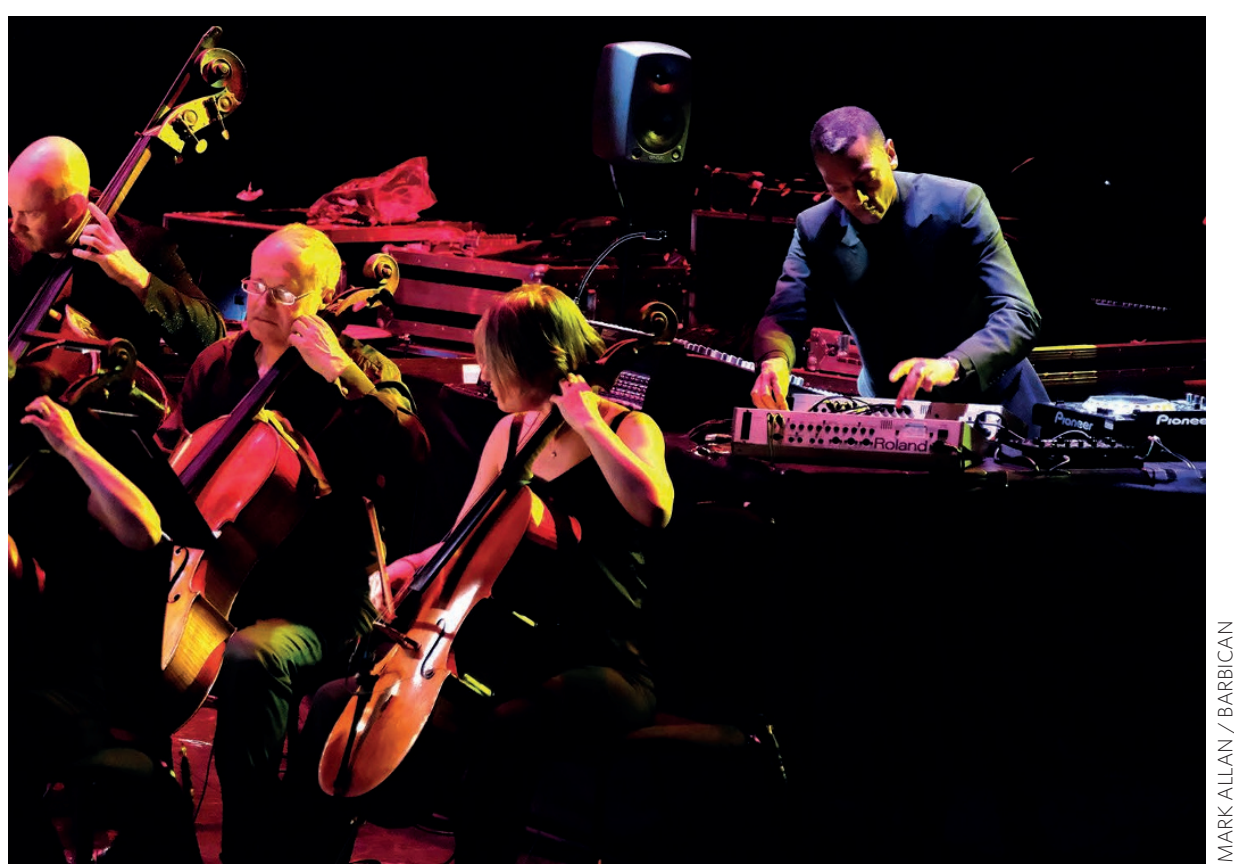

impossible to avoid associating the two sets of instruments to the old and the new, and perhaps the composer played with this idea too.

Like new data do to our previous theories of space, sometimes the bass quietly fills the gap left by the classical music. Sometimes the beats strengthen the melody, accelerating it with a space-race-style sensation. In other instances, the electronic sounds take over, dominating the symphony - particularly for the more distant planets and the inter-planet transits.

But this should not come as a surprise. In the ten years spent on this project, Mills did not limit himself in his search for musical inspiration. Rather, he sifted through data from NASA and other space agencies to incorporate such information in his music. Mills looked into how humans travel through space, and how planets compare against each other in terms of rotation speed, chemical composition and water content - all in an effort to make his composition as close to actual data as possible.

Despite the scientific framework in which Planets has been constructed, it's hard to shake off the feeling that the romantic idea of space in Holst's sense is still there, somewhere. Space does have its very own pull on human hearts, after all. The transit between the Earth and
Mars is full of a sense of expectation, almost making us wonder when we will get there in reality. At the start of Jupiter's track, a lonely trumpet gives a feeling of warmth and sweet homeliness, maybe what one would feel after having crossed the asteroid belt in the long journey from Mars. And right before we arrive at Saturn, the orchestra scatters through the auditorium - with trombones and tubas at the two opposite corners of the hall, and trumpets on the upper floors - perhaps suggesting that there is more to Saturn than just the planet's surface.

The pursuit for knowledge that contributed to the transformation of Holst's 1918 The Planets into Mills's 2015 Planets does not always follow a straight path. The artists decided to acknowledge the uncertainty that underpins all scientific efforts by opening the evening with The Unanswered Question by American composer Charles Ives, a peculiar piece involving a back-and-forth between trumpets and woodwind against a backdrop of strings. This musical Q\&A session gets increasingly frustrated and dissonant - as if the instruments were speaking different languages. Perhaps showing NASA data to an astronomer from 1918 would have the same effect.

REVIEWED BY FEDERICO LEVI 